\title{
Verbesserung der Temperaturmessauflösung durch SuperResolution bei handgehaltenen Wärmebildkameras
}

\section{Getting the Most out of a Few Pixels}

\author{
Autoren: \\ Evers-Senne, Jan-Friso; Schmieder, Matthias; Stratmann, Martin \\ Testo AG, Celsiusstraße $2 \cdot 79822$ Titisee-Neustadt • Germany \\ +49 7653681 -0, \{ JFEversSenne | MSchmieder | MStratmann \}@testo.de
}

\section{Abstract}

Today's thermal imagers have a very limited spatial resolution. This article analyses the deficiencies and proposes to use a system called SuperResolution to enhance the spatial resolution. SuperResolution combines de-convolution with super-sampling and therefor addresses the two main limitations of IR-cameras. SuperResolution works by capturing multiple images of a hand-held camera quickly, and then computing a high resolution image from them. Finally, the SuperResolution approach is analyzed in detail, to verify the effective resolution enhancement and to ensure that temperature values derived from the final images are correct.

\section{Motivation}

Thermal imaging is a key technology for building insulation inspections and predictive maintenance of both mechanical and electrical systems. State-of-the-art thermal imagers make use of uncooled micro-bolometer FPAs. Sensor resolutions range form 160x120 and below up to $640 \times 480$ and in rare cases up to $1024 \times 768$. Pixel sizes vary between $25 \mu \mathrm{m}$ which is standard today and $17 \mu \mathrm{m}$ for newer sensors. The majority of thermal imagers are sensitive in the LWIR $(8-14 \mu \mathrm{m})$ for special applications the MWIR $(3-5 \mu \mathrm{m})$ is also used.

The pixel resolution frequently limits temperature measurements on small objects. Getting closer to the object is e.g. in case of electrical high-voltage installations not always possible.

The only solutions today are to change the lens and loose flexibility or spend lots of money for a high resolution camera.

Our claim here is to present an alternative approach: Increase the camera resolution by using a combined computation of de-convolution and super-sampling. Our approach allows addressing the two key factors that limit IR-camera resolution: a) Blurring by lens aberrations and diffraction and b) poor sampling due to limited detector resolution.

Fig 1 shows the difference between the super-resolution approach and a classical interpolation. The interpolation cannot create more details in an image. The SuperResolution approach is capable to recover image details that are lost in a single frame due to image blur and detector sampling.

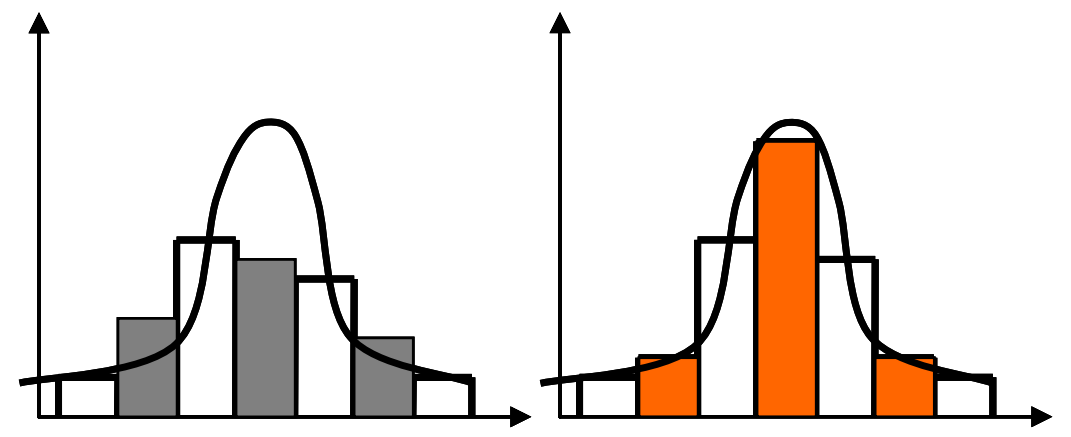

Figure 1:Solid line represents ideal signal. White columns show measurement values sampled with detector resolution. Left figure: Grey columns are obtained by classical interpolation: peaks 


\section{Principles and Building Blocks of SuperResolution}

\section{Signal Chain}

Using an infrared-camera, the user observes a certain region of the world. The signal emitted from the observed object is continuous in space and time by its nature. This signal passes through different components of the IR-camera and is modified on its way to the observer. Figure 2 illustrates this signal chain which is described in more detail now. In the following it is assumed that the complete setup is stationary and does not change over time. So, all timevarying-aspects can be ignored.

The object is assumed to be a hot wire in front of a cold background. The corresponding continuous signal is plotted below the object as a rectangular signal with infinite steep edges. When passing through the lens and the aperture, this signal is blurred to something similar to a Gaussian distribution function (see the following section for more details).

The blurred signal hits the IR-detector consisting of several discrete micro-bolometers. The sensor integrates the signal over the area of each micro-bolometer and thus quantifies the signal in space resulting in a step-function.

Finally, the camera electronics and firmware modifies this discrete signal to compute temperature values and to produce a visual appealing image for the observer. The observer can see a colour-code version of the discrete signal with each colour representing a certain temperature.

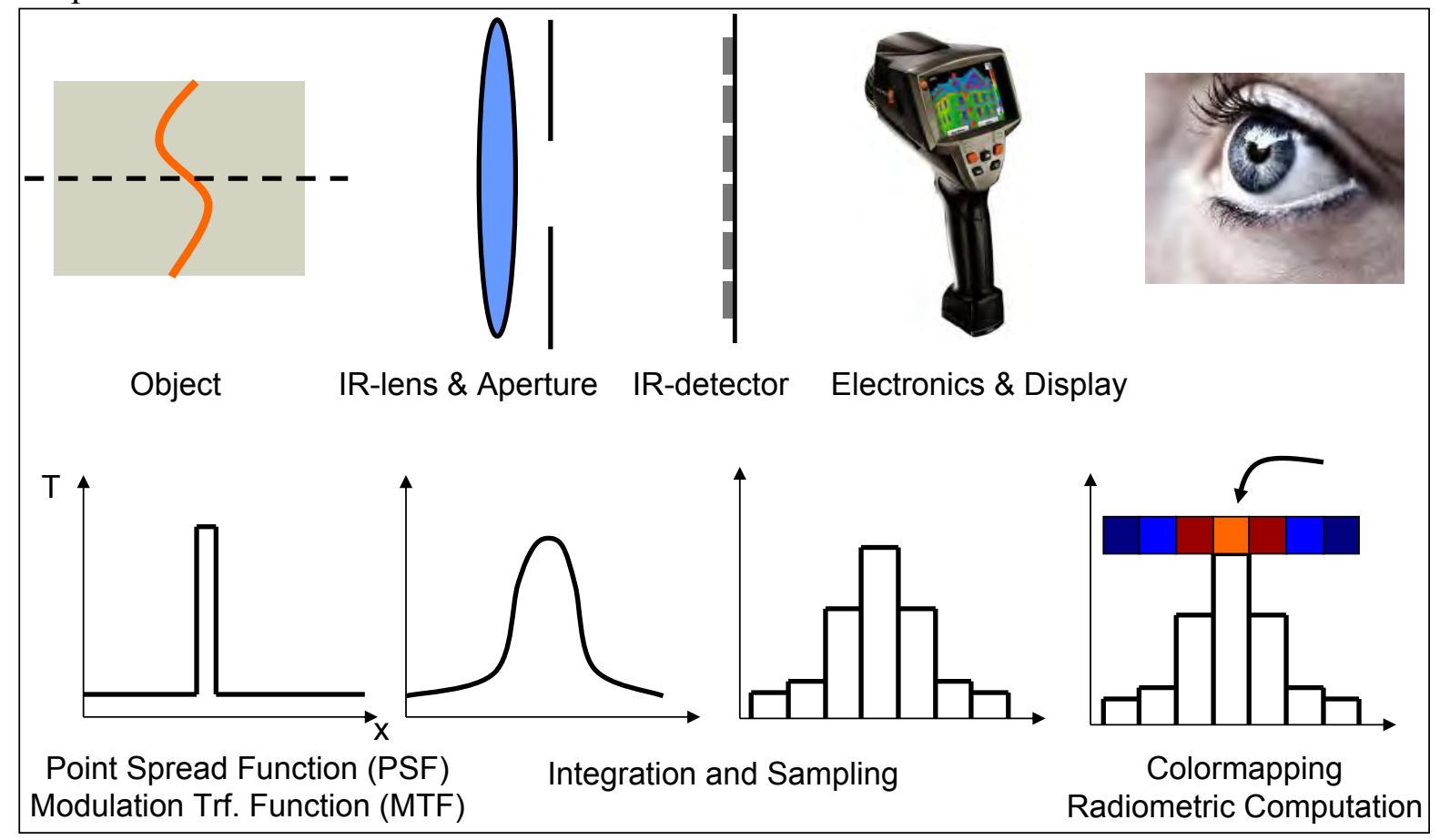

Figure 2: Signal chain of an IR-camera and how the signal is modified.

\section{Point Spread Function}

The optical path of an IR-camera consists at least of one or more lenses and an aperture. All light travelling through this system is modified by different effects, most notably chromatic aberration and diffraction.

Due to the fact that the refraction index of most materials is a function of wavelengths, and that the usual LWIR spectrum ranges from 8 to $14 \mu \mathrm{m}$, rays of different parts of the spectrum are 
refracted differently. This is called chromatic aberration and leads to some amount of blur of each point observed by a camera.

The diffraction also depends on the wavelength and is defined by the so called Airy disc. The Airy disc is the 2-dimensional distribution function of Dirac-impulse of light at a given wavelength when travelling through an aperture. Figure 3 left illustrates the Airy disc. One approach to quantify the amount of blur introduced by an optical system is the so called Point-Spread-Function (PSF). It summarises the contributions of all effects without the need to analyse each effect in detail. The point-spread-function describes the resulting signal for an input signal of a very short peak (Dirac-impulse). This is equivalent to the impulse response function for one-dimensional signals. An example of the point-spread-function is shown in Figure 3 right.
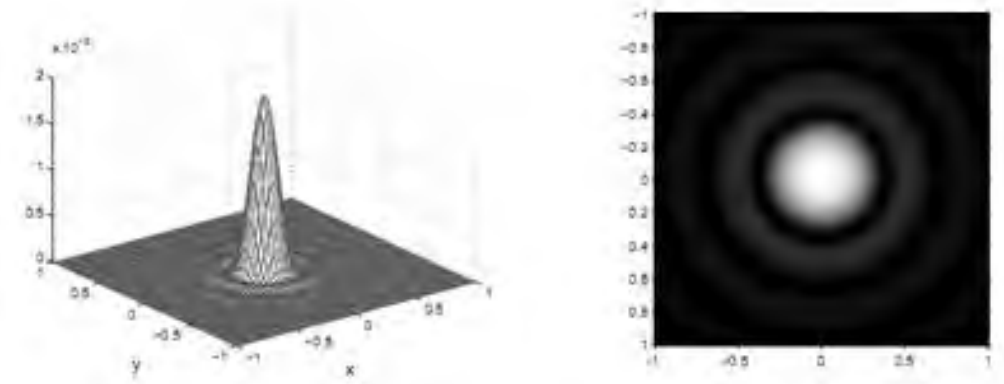

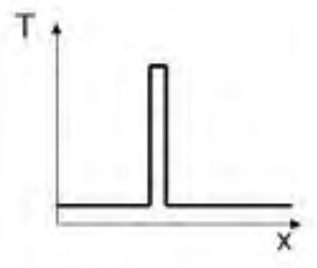

Input

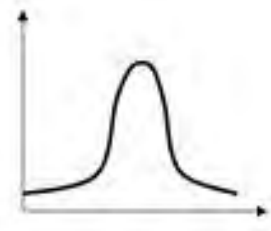

Output

Figure 3: Left: The Airy-Disc is the 2-dimensional impulse response of an aperture. Right: The point spread function describes how a small spot is spread over an area.

\section{De-Convolution}

The point-spread-function can be simulated with a simple FIR filter by using a Gaussian filter mask. A discrete signal convolved with a Gaussian distribution function results in a signal where the energy is distributed over an area. This process cannot simply be inverted, because the convolution is not invertible.

The process of de-convolution is an iterative process which tries to find an original signal which results in the observed signal when convolved with a given PSF. If even the PSF is unknown, this can also be estimated by the so-called blind de-convolution. But this is a computational expensive task and several prerequisites have to be fulfilled.

From the application view, the de-convolution tries "re-focus" the distributed energy thus sharpening the image. It cannot reconstruct small details which got lost by the detector sampling.

\section{Pixel Pitch and Super-Sampling}

After passing the optical system, the signal is still a continuous one. The detector with its pixel structure discretises the signal in space. In Figure 4 (left) a small part of a detector with four bolometers is shown. Each bolometer contributes one pixel to the final image. Due to mechanical limitations, the sensitive area of a micro-bolometer is not identical to the space it requires on the focal plane. This space is given by the pixel pitch. Only a fraction of about $75-85 \%$ of the squared pixel pitch is sensitive to radiation (fill-factor).

When sampling a continuous signal with such a detector, the highest possible frequency which can be sampled and reproduced (Nyquist frequency) is half of the sampling frequency. But the sampling frequency is not determined by the size of the sensitive area but by the pixel pitch containing also the blind regions. Thus, the continuous signal must be band-limited to the Nyquist-frequency or aliasing will occur otherwise. That means, that even an ideal optical system in front of the detector will not allow increasing the spatial resolution above the pixel pitch. 


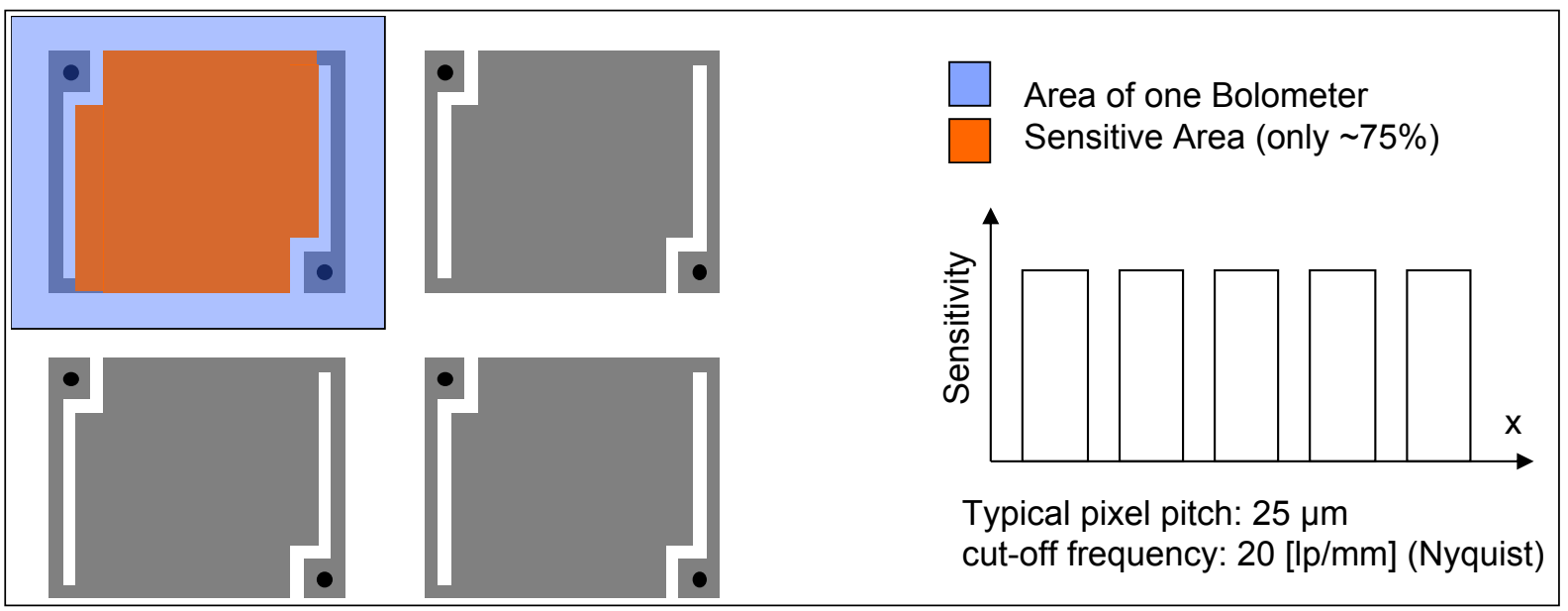

Figure 4: Sampling of an IR-detector. Each micro-bolometer integrates over an area an a time period.

By introducing super-sampling, the resolution limit can be pushed beyond the pixel pitch. The basic idea is to take several images of a given object from positions shifted by $1 / 2$ pixel pitch as shown in Figure 5. This "fills the gaps" between the bolometers by a second image and double the sampling frequency (or halve the pixel pitch). By using more images shifted by $1 / 3$ or $1 / 4$ of the pixel pitch, an even higher sampling frequency can be reached theoretically. This allows reconstructing small details which are otherwise beyond the Nyquist-frequency.

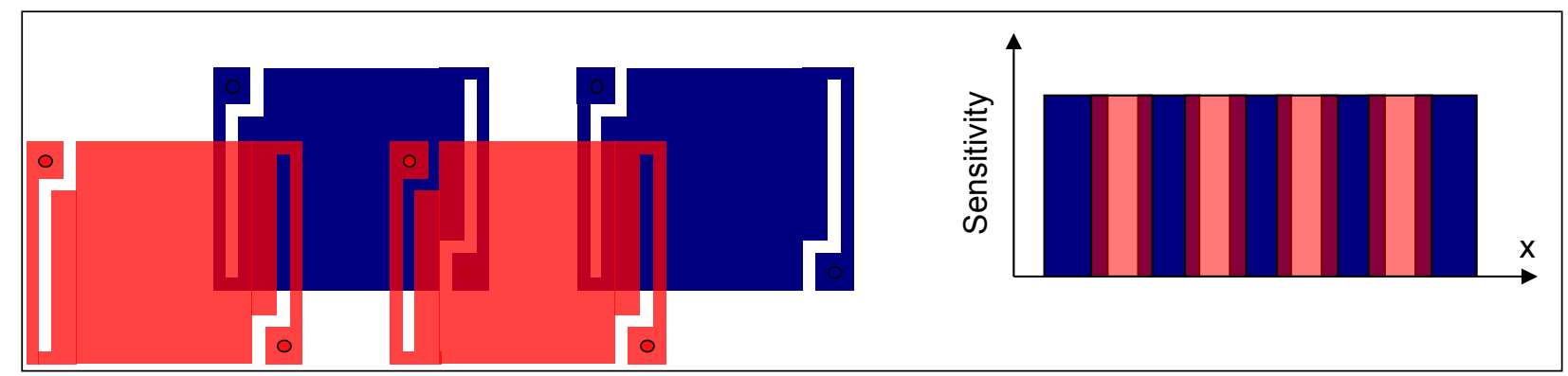

Figure 5: With super-sampling, multiple shifted images are used to place more sample.

\section{Implementation}

Both image enhancements, namely super-sampling and de-convolution, already exists for IRcameras. But neither really gave the promised results. Super-sampling alone suffers from uncompensated PSF-blur and cannot reconstruct small details which are already lost in the optical system. De-convolution alone greatly enhances the visual appearance of an image, but the limitation to the sampling frequency of the detector prohibits the reconstruction of small details. Only the combination of both approaches can really increase the spatial resolution of the imaging device. In (1) this has been demonstrated for images in the visual spectrum.

We adapted the approach from Sroubek et al. together with the original authors to fulfil our special needs. Our contribution here is that we

- optimised the implementation for the special use in combination with our IR-cameras,

- verified that the measured temperatures are accurate and

- quantified the achieved resolution enhancement.

This effort makes the proposed system usable for anybody.

\section{Image Registration}

Instead of moving the camera, the sensor, or the optical path mechanically, we decided to use the natural tremor of the user as actuator. It is sufficient to move the camera by a few micrometres, 
and for a hand-held camera this can always be assumed. But obviously we cannot restrict the user to such small movements. Neither do we know the real movement. So our SuperResolution captures a number of images in a short time period (typically in $1 / 5$ second), each shifted randomly by the users movement.

The processing starts by registering the images against each other. This registration process uses image processing algorithms to compute the movement between each image pair. In a first step, rough motion estimation computes the movement only with accuracy up to one pixel. In a following fine registration, the movement between two images is refined with sub-pixel accuracy.

\section{Image Reconstruction}

The image reconstruction step combines the information from the registered multiple images into a new image of increased resolution. Because no direct transformation from a low resolution image to a high resolution image exists which is able to reconstruct small details, an iterative approach is chosen.

Image degradation from a high resolution image $I_{h}$ to one or more low resolution images $I_{k}$ can be described as a sequence of simple image transformations:

$$
I_{k}=D\left(u * s_{k} * I_{h}\right)+n
$$

With $s_{k}$ a shift-operator simulating the camera movement, $u$ the expected blur (PSF), D() a decimation operation which reduces the number of pixels by re-sampling, and $n$ a portion of additive noise. The *-operator denotes a convolution.

In the original implementation according to (1), the blur $u$ is expected to be volatile, thus it changes between different recordings and has to be estimated together with the high-resolution image itself. In our implementation we assume that $u$ is constant for a given camera and thus can be determined beforehand. The expected noise has to be modeled explicitly to avoid amplifying it during image processing. The term $s_{k}$ is initialized with the shift computed in the image registration process and the decimation operation $D()$ is known as the ratio between the size of the low resolution images and the size of the high resolution image.

Based on this image formation process, a set of $K$ low resolution images gives exactly $K$ such equations forming an equation system with one unknown variable: $I_{h}$. A solution for this system can be found by minimizing the differences between the transformed and decimated high resolution image(s) and the real low resolution images. This iterative process modifies the $I_{h}$, such that the differences between all real images and the associated transformed images become minimal.

The iteration starts by initializing $I_{h}$ with an up-sampled (interpolated) version of one low resolution image $I_{k}$. Using a gradient descend algorithm, pixels values of $I_{h}$ are then modified, and the result is evaluated by computing the difference between the simulated low resolution images and the real images. This modify/verify-scheme is then repeated until a given minimal change is reached.

\section{Prerequisites}

The presented combination of de-convolution and super-sampling does not require any special hardware. It only needs several images taken by a hand-held IR-camera in a short period of time. This allows us to offer this feature not only for new IR-cameras but for nearly all our IR-cameras on the market. In technical terms, our so called SuperResolution feature is only a software update. 


\section{Verification and Results}

\section{Spatial Resolution}

The spatial resolution of an imaging device can be specified as Modulation Transfer Function (MTF), sometimes also referred as Optical Transfer Function. The MTF is a function which defines the relative contrast over the input frequency in [line-pairs / mm]. For low spatial frequencies the relative contrast is typically near 1.0 and it decreases for higher frequencies.
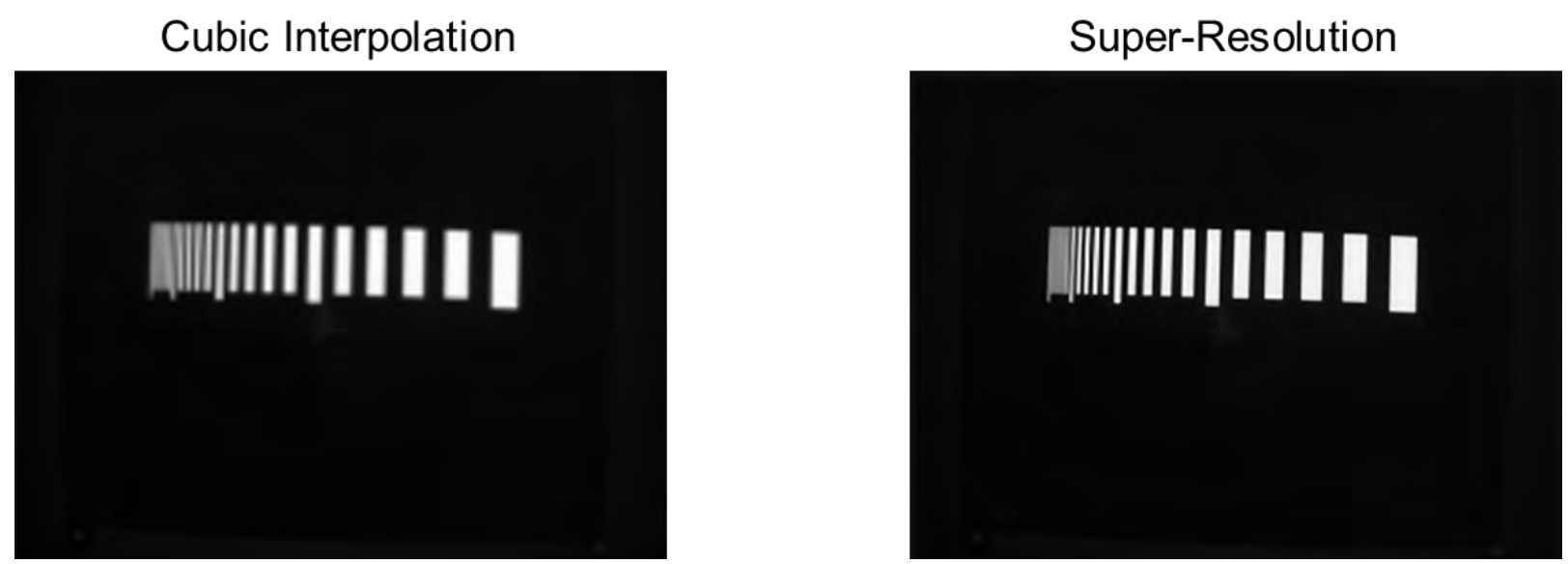

Figure 6: Two images of the Variable Frequency Bar Target. The spatial resolution increases from right to left.

The MTF of an infrared imaging device can be measured with a pattern which shows different frequencies like the Variable Frequency Bar Target used here. The camera is placed in a given distance in front of the target and the captured images are analyzed along a horizontal line crossing the pattern. This gives an intensity signal as shown in Figure 7.

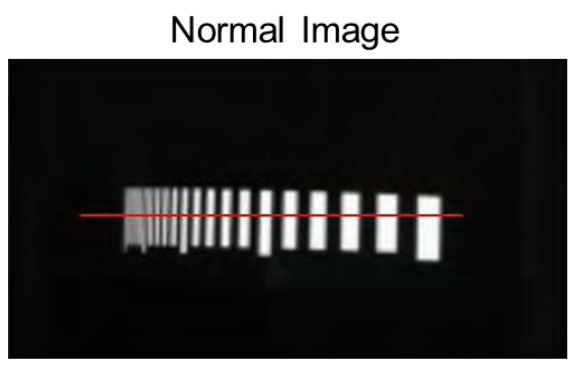

Superresolution

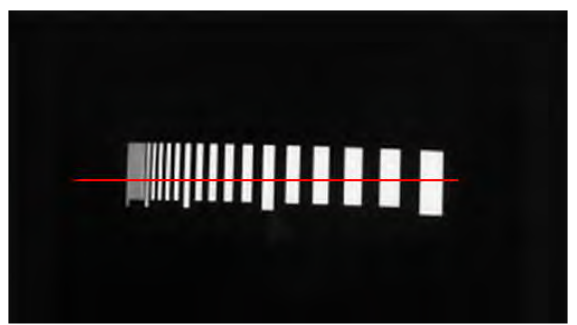

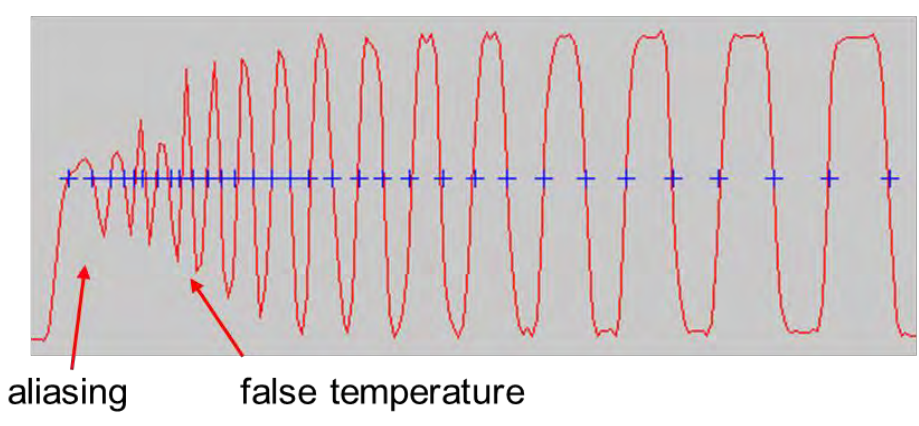

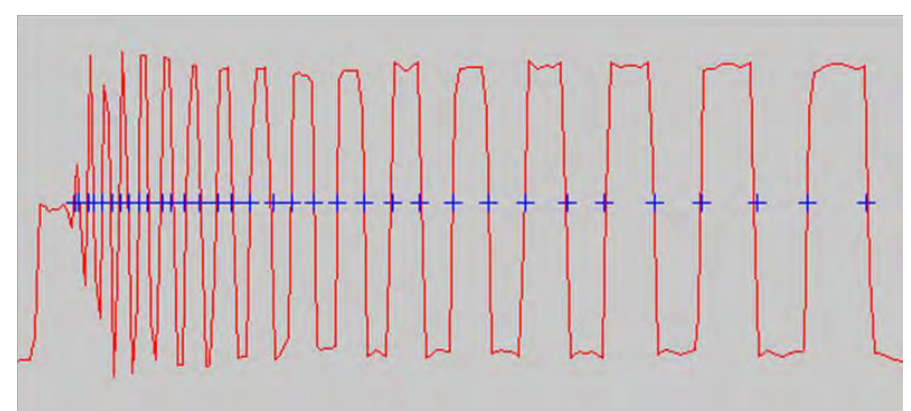

Figure 7: intensity signal along the red line.

The signal can then be transformed to spatial frequencies using the pixel pitch of the detector for a normal image. For the SuperResolution image the pixel pitch is assumed to be half of the sensor pixel pitch due to the doubled image dimensions. In Figure 8 the resulting MTF is shown. To allow the characterization of optical systems with one single number, we choose the spatial frequency for which the relative contrast is $50 \%$. Based on these numbers, the spatial resolution 
of the Testo 881 is $15[\mathrm{lp} / \mathrm{mm}]$ and with SuperResolution this can be increased to $24[\mathrm{lp} / \mathrm{mm}]$. This gives a resolution enhancement of factor 1.6.

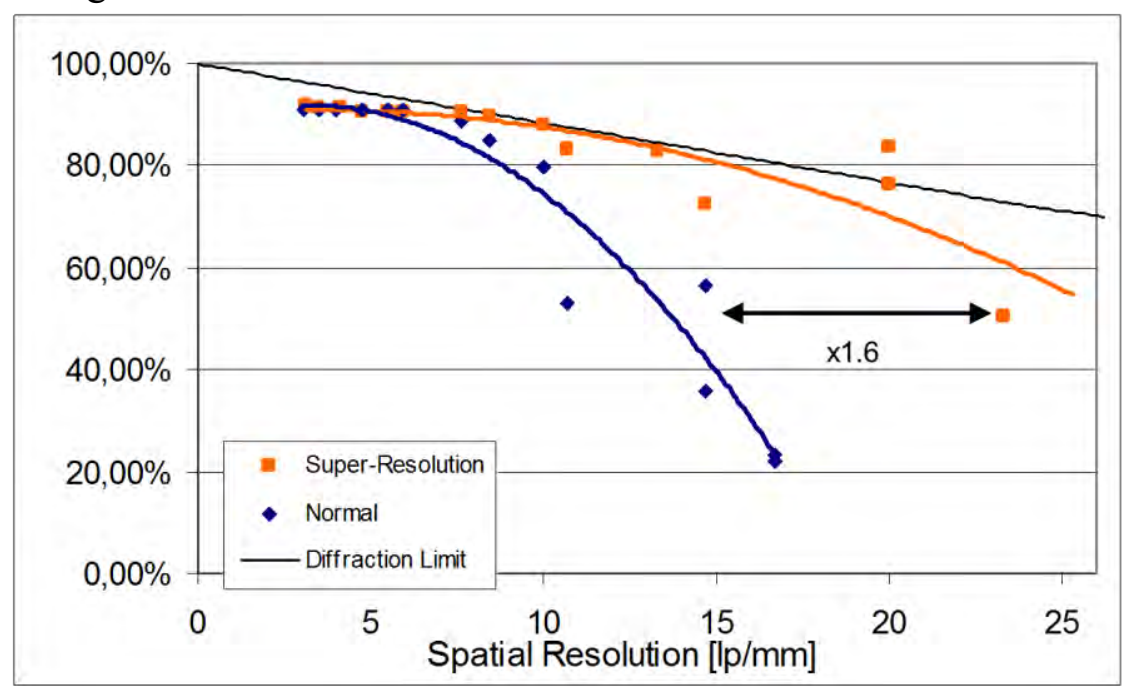

Figure 8: MTF for a Testo $881,160 \times 120$, pixel pitch $25 \mu \mathrm{m}, \mathrm{F} 0.84$

\section{Smallest Measurable Object}

The MTF as discussed above only evaluates the relative contrast. It does not take the absolute values into account. For a measurement device like a calibrated infrared imager the absolute values are even more important. To evaluate the influence of SuperResolution to the measurement of objects of different size, a setup with a variable aperture is used.

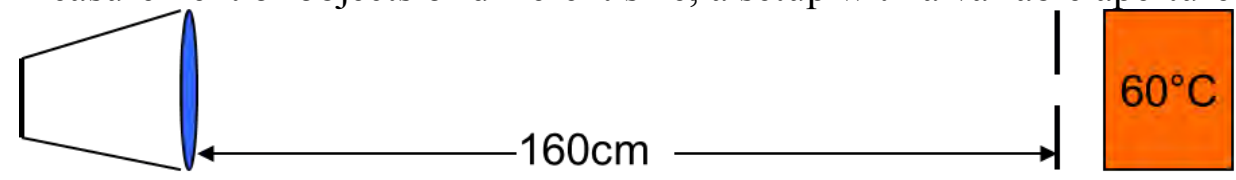

Figure 9: An infrared camera looking at a variable aperture in front of a black body radiator.

The distance to the aperture is $1.6 \mathrm{~m}$, the temperature of the radiator is set to $60^{\circ} \mathrm{C}$ and the diameter of the aperture can be varied between $2 \mathrm{~mm}$ and $32 \mathrm{~mm}$. Figure 9 depicts the situation, while Figure 10 shows two images as seen by the camera.

The Min-Max-on-Area function is the used to determine the measurable temperature as observed by the camera. By varying the diameter of the aperture, the temperatures for objects of different sizes are recorded. Figure 11 shows a plot of these values for a Testo 885 with 320x 240 pixels and a pixel pitch of $25 \mu \mathrm{m}$ using a standard lens $\left(32^{\circ}\right)$.

Without SuperResolution (grey curve), the observed temperature decreases with decreasing object size. The theoretical limit for the object as specified to be measured correctly is $7.8 \mathrm{~mm}$ and infact, for $8 \mathrm{~mm}$ the observed temperature is only $57.5^{\circ} \mathrm{C}$.

With SuperResolution (orange curve), all measured temperatures are slightly above the values without SuperResolution (see next Section for details about this effect). Assuming a resolution gain of 1.6, the theoretical limit for the smallest measurable object is $7.8 \mathrm{~mm} / 1.6=4.8 \mathrm{~mm}$. For $6 \mathrm{~mm}$ diameter, the measured temperature is $60.7^{\circ} \mathrm{C}$, for $4 \mathrm{~mm}$ it is 62.5 , thus the expected temperature for $4.8 \mathrm{~mm}$ is in between. 

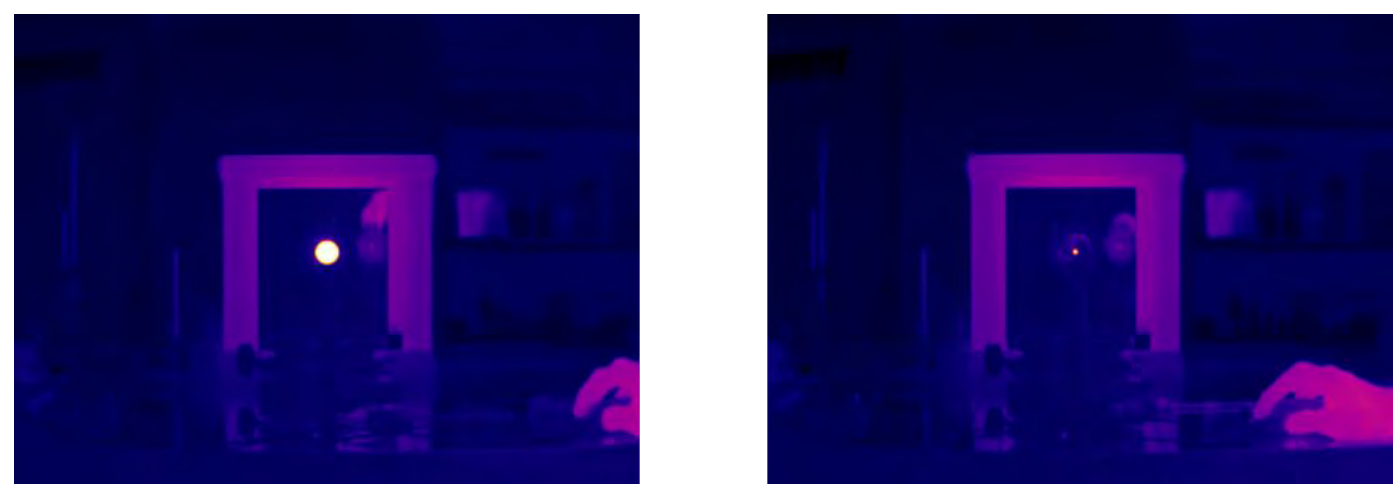

Figure 10: Images form the infrared camera observing the variable aperture. Left: Diameter=32mm, right: diamater $=6 \mathrm{~mm}$

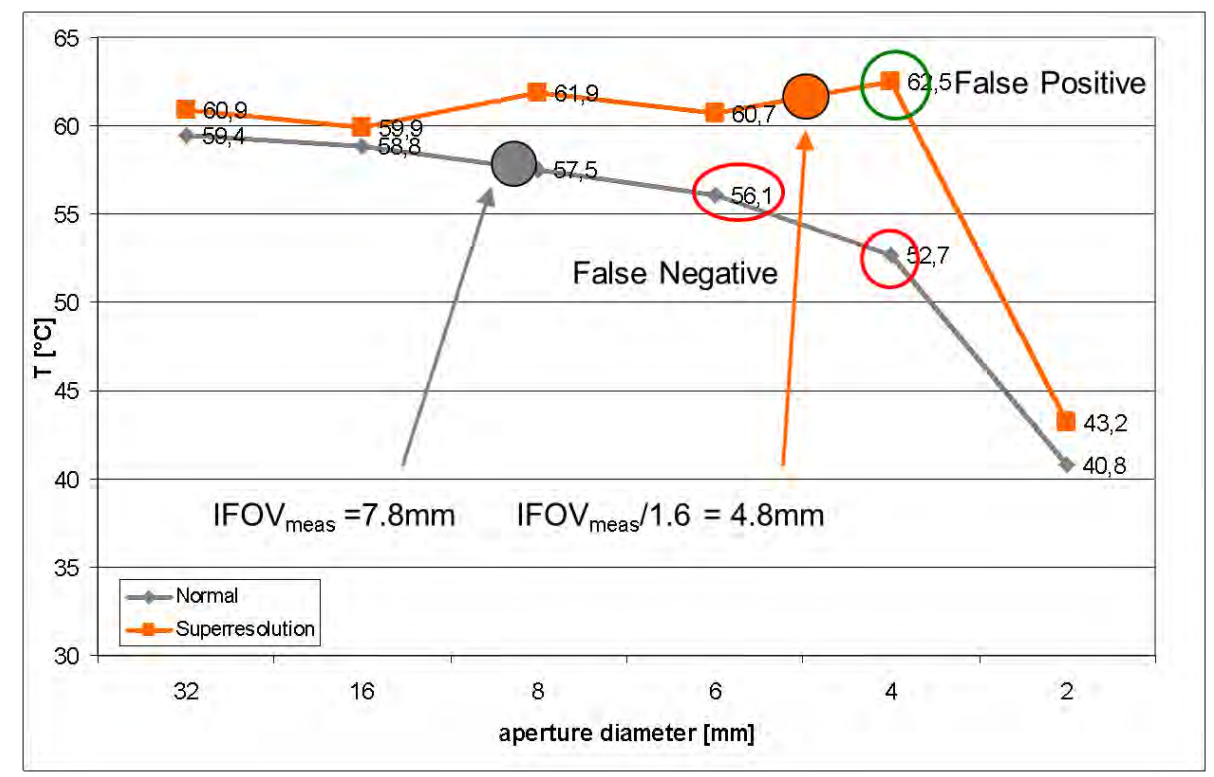

Figure 11: $320 \times 240$ pixel, $25 \mu \mathrm{m}$ pixel pitch, $32^{\circ}$-Lens, Distance $=160 \mathrm{~cm}$

The object size of $4 \mathrm{~mm}$ is below the theoretical limit of $4.8 \mathrm{~mm}$, and as clearly visible, the observed temperature exceeds the tolerance of $+/-2^{\circ} \mathrm{C}$. But even for that object size the measured value is not smaller than the reference $\left(60^{\circ} \mathrm{C}\right)$ but greater. In other words, in case of a hot object of smaller size then the limit, the operator will get a false positive instead of a false negative without SuperResolution. For even smaller objects like the aperture of $2 \mathrm{~mm}$ diameter, the observed temperature drops similar to the measurement without SuperResolution.

\section{Modifications to the Signal}

The SuperResolution applies signal processing algorithms to the infrared images and therefore modifies the temperatures measured. Figure 12 shows the signals from a $32 \mathrm{~mm}$-aperture and a $4 \mathrm{~mm}$-aperture both with and without SuperResolution. The blue plot is the signal of the unprocessed image of an object which smaller then the theoretical limit $(4 \mathrm{~mm})$. The peak temperature is about $52^{\circ} \mathrm{C}$ which is only measured at one pixel, the neighboring pixels are at $30^{\circ} \mathrm{C}$. The signal processing of the SuperResolution (green plot) adds more pixel and at the same time enhances the contrast of the edges. The values at $x=8$ and $x=9$ are reduced while the values at $\mathrm{x}=10,11$ and 12 are increased resulting in a reconstruction of the real temperature of $60^{\circ} \mathrm{C}$. The same effects occur for signals from larger objects, the black line belongs to a diameter of $32 \mathrm{~mm}$ without SuperResolution. After processing the images with the SuperResolution algorithm, the edges are steeper and before and after an edge, a small overshoot is created. All values inside the homogenous region of the object stay the same. 
So taking the temperature with cursor in the center of an object yields the correct temperature, while the maximum value reported is slightly greater.

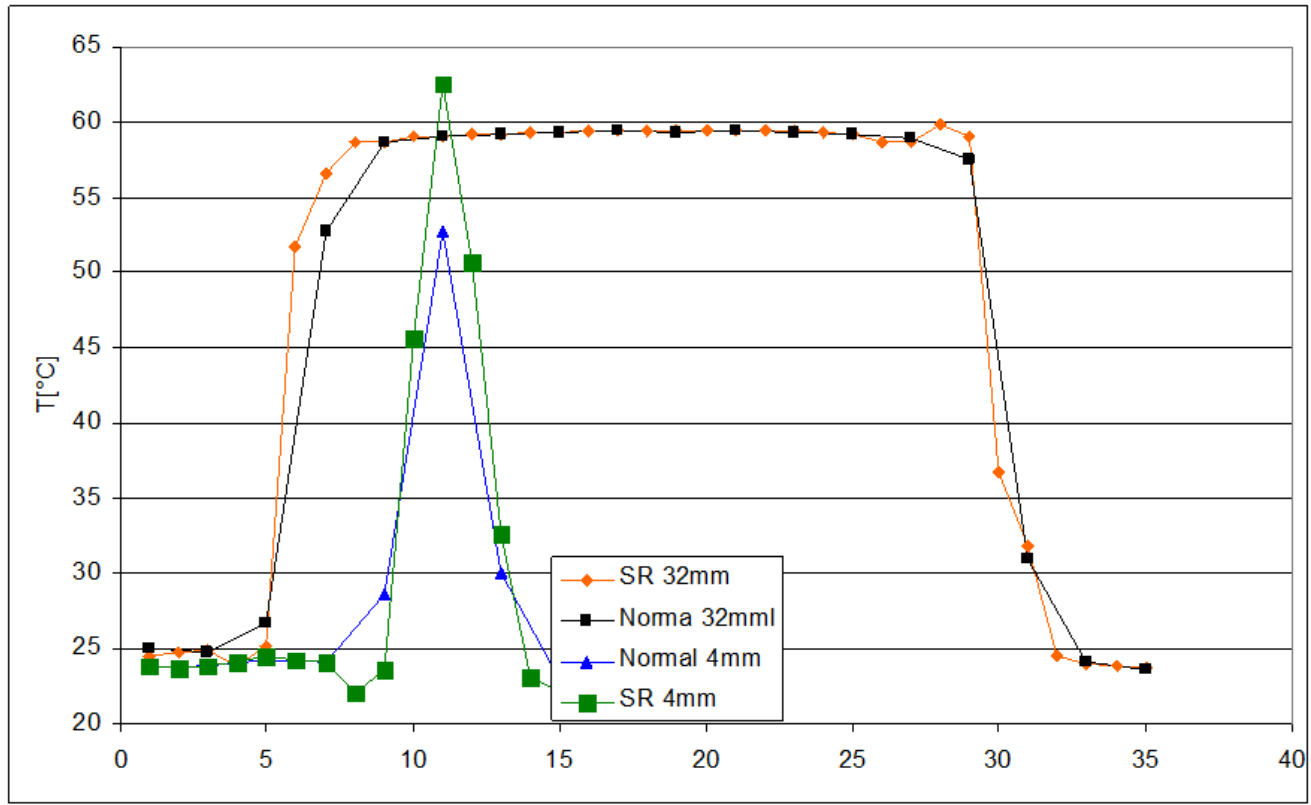

Figure 12: Temperature signals (one row of pixel from an image) of a 32mm-aperture( black, orange) and a $4 \mathrm{~mm}$-aperture (blue, green).

\section{Conclusion}

We presented a specialized approach to enhance the spatial resolution of an IR-camera with a standard micro-bolometer FPA by combining de-convolution with super-sampling. While the deconvolution addresses the blur of the optical path, super-sampling helps to overcome the limitations of the pixel grid. We optimized the implementation by applying several assumptions for the IR-cameras. Finally we analysed the modifications to the image signal and measured an effective resolution gain of 1.6.

The system presented here is commercially available and can be used in conjunction with our Ircameras.

\section{References}

1. Sroubek, F., Cristobal, G. und Flusser, J. A Unified Approach to Superresolution and Multichannel Blind Deconvolution. IEEE Transactions of Image Processing. Sept, 2007, Bd. 19, 9. 